\title{
Holobiont Evolution: Mathematical Model with Vertical vs. Horizontal Microbiome Transmission
}

\author{
Joan Roughgarden ${ }^{* \dagger}$
}

\begin{abstract}
A holobiont is a composite organism consisting of a host together with its microbiome, such as a coral with its zooxanthellae. To explain the often intimate integration between hosts and their microbiomes, some investigators contend that selection operates on holobionts as a unit and view the microbiome's genes as extending the host's nuclear genome to jointly comprise a hologenome. Because vertical transmission of microbiomes is uncommon, other investigators contend that holobiont selection cannot be effective because a holobiont's microbiome is an acquired condition rather than an inherited trait. This disagreement invites a simple mathematical model to see how holobiont selection might operate and to assess its plausibility as an evolutionary force. This paper presents two variants of such a model. In one variant, juvenile hosts obtain microbiomes from their parents (vertical transmission). In the other variant, microbiomes of juvenile hosts are assembled from source pools containing the combined microbiomes of all parents (horizontal transmission). According to both variants, holobiont selection indeed causes evolutionary change in holobiont traits. Therefore, holobiont selection is plausibly an effective evolutionary force with either mode of microbiome transmission. The modeling employs two distinct concepts of inheritance, depending on the mode of microbiome transmission: collective inheritance whereby juveniles inherit a sample of the collected genomes from all parents, as contrasted with lineal inheritance whereby juveniles inherit the genomes from only their own parents. A distinction between collective and lineal inheritance also features in theories of multilevel selection.
\end{abstract}

\section{Keywords}

holobiont $\bullet$ holobiont selection $\bullet$ hologenome $\bullet$ microbiome $\bullet$ multilevel selection $\bullet$ collective inheritance $\cdot$ lineal inheritance

*Department of Biology, Stanford University, Stanford CA 94305, USA, joan.roughgarden@stanford.edu ${ }^{\dagger}$ Hawaii Institute of Marine Biology, University of Hawaii, Kaneohe HI 96744, USA

(D) https://orcid.org/0000-0002-6360-7964

Received 7 July 2019; Revised 21 October 2019; Accepted 23 October 2019

doi:10.3998/ptpbio.16039257.0012.002 


\section{Introduction}

This paper develops a simple, conceptual, mathematical model for the evolution of holobionts. Its purpose is to clarify how holobiont selection may cause evolutionary change in the traits of holobionts.

A microbiome is an "ecological community of commensal, symbiotic, and pathogenic microorganisms" that shares the "body space" of a host (Lederberg and McCray 2001). A bolobiont is a composite organism consisting of a host together with its microbiome (Margulis 1991). The bologenome is the union of all the host genes with all the genes in its microbiome (ZilberRosenberg and Rosenberg 2008). To these definitions I add the following: a bologenotype is the configuration of the hologenome in an individual holobiont. The bologene pool is the set of all hologenotypes in a population of holobionts. A holophenotype is a hologenotype's expression in the behavior, physiology, and morphology of a holobiont. Holobiont selection is the differential reproduction and/or survival of holobionts as a function of their holophenotypes. The hologene pool is represented by the total holobiont population size together with a frequency distribution of hologenotypes over the space of all possible hologenotypes. Through time, holobiont selection changes both the total number of holobionts as well as the frequency distribution of hologenotypes.

Many researchers have suggested that selection operates on the combination of the host together with its microbiome as a unit (Woese 2004; Rosenberg et al. 2007; Fraune and Bosch 2010; Guerrero, Margulis, and Berlanga 2013; Gilbert, Sapp and Tauber 2012; McFall-Ngai et al. 2013; Rosenberg and Zilber-Rosenberg 2014; Bordenstein and Theis 2015; Lloyd 2017; Rosenberg and Zilber-Rosenberg 2018). According to this perspective, the union of a host's genes together with the genes from all the microbes living in it comprises an extended genotype, called here the hologenotype. Selection on holobionts then changes hologenotype frequencies in a population of holobionts with a process envisioned as similar to how classic natural selection on individuals changes genotype frequencies in a population of individuals. Proponents of holobiont selection have documented astonishingly intricate and intimate integration between hosts and their microbiomes in behavior, physiology, anatomy, and development and argue that this integration itself is evidence of the power of holobiont selection to produce host/microbiome coadaptation.

Other researchers are unconvinced that holobiont selection actually accomplishes the evolutionary results claimed for it (Booth 2014; Moran and Sloan 2015; Douglas and Werren 2016; Hester et al. 2015; Skillings 2016; Queller and Strassmann 2016; Doolittle and Booth 2017). They observe that vertical transmission of microbiomes from parental hosts to juvenile hosts is uncommon. Instead, juvenile hosts usually acquire microbiomes from the surrounding environment, leading to horizontal transmission. Hence, the microbiome in a holobiont is an acquired trait. Because the microbiome is not inherited, holobiont selection presumably cannot cause holobiont evolution.

The skeptics acknowledge the existence of host/microbiome integration, but deny that holobiont selection accounts for the evolution of this integration. They note further that microbiomes are not necessarily helpful to the host, but can contain pathogens that hurt the host. They argue that a balanced view of the host/microbiome relation would include more mention of deleterious microbes to supplement the mutualistic microbes emphasized by the holobiont selection proponents.

The response of holobiont selection proponents to the objections raised by the skeptics is to demonstrate that vertical transmission is actually more common that the skeptics claim. The microbiome does not need to be contained in the gametes of the parental host to be considered 
as being transmitted vertically. The parental host's microbiome may colonize (or infect) the juveniles during birth, for example during passage through the birth canal in mammals, or from contact with nest material in birds, and so forth.

Although the skeptics may underestimate the amount of vertical transmission of microbiomes, this response by the proponents still remains unsatisfying. For example, in a few species of corals the zooxanthellae are transmitted vertically in the coral's gametes (Hirose and Hidaka 2006). But the vast majority of zooxanthellae are acquired from the open sea water surrounding the coral (Babcock et al. 1986; Trench 1993). Similarly, vertical transmission of the microbiome in sponges is described as inconsistent and unfaithful (Björk et al. 2019). Although both these examples are marine, even terrestrial groups may offer less vertical transmission than might be expected. In a live-bearing cockroach, researchers found only one component of the microbiome to be transmitted vertically, a bacterium that apparently supplies nutrients needed during development (Jennings 2019). But they also found that a majority of the microbiome components are acquired from the environment throughout the first-instar and melanization period (Jennings, Korthauer, Hamilton, and Benoit 2019). So, the limited vertical transmission of the microbiome in many types of holobionts remains a difficult point for holobiont selection proponents.

On the other hand, the holobiont selection skeptics face the reality that extensive and intricate host/microbiome integration has indeed evolved somehow. The skeptics argue that classic coevolutionary selection operating on separate, yet interacting, host and microbiome genotypes can produce that integration. It is well known that coevolutionary selection has led to many coadaptations between macro-organisms such as the classic plant-pollinator and damselfishsea anemone associations, among others. Coevolutionary selection has also produced intricate schemes by which hosts reduce parasite virulence involving both chemical mimicry and geographically located arms races (Nash, Als, Maile, Jones, and Boomsma 2008). Furthermore, both horizontal and vertical pathogen transmission leading to the coevolution of reduced virulence by hosts has been studied theoretically (Yamamura 1993; Lipsitch, Siller, and Nowak 1996). Still, the degree of integration between host and microbiome is claimed by holobiont proponents to be of an entirely different scale than that seen in the known examples of coevolutionary coadaptation. This opinion, however, is subjective and skeptics can assert that given enough time, classic coevolutionary selection will eventually yield the degree of host/microbiome integration highlighted by the proponents.

So, at this time, two quite reasonable alternative positions occur with respect to holobiont selection. The proponents face the difficulty of dealing with the largely horizontal mode of microbiome transmission. The skeptics must insist that classic coevolutionary selection is sufficient to produce the deeply integrated coadaptation that occurs in the host/microbiome relation.

In this context, a simple conceptual model might help clarify how holobiont evolution can be attained with holobiont selection. This article specifically addresses the question of microbiome transmission. It analyzes whether holobiont evolution via holobiont selection is plausible depending on the mode of microbiome transmission. This study responds to the demand from medical and conservation constituencies for understanding the microbiome/host relationship (Turnbaugh et al. 2007; Dubilier, McFall-Ngai, and Zhao 2015; OSTP 2016).

The models introduced here are intentionally simplistic. Many review articles document an extraordinary complexity of microbiomes across diverse systems in mammals, plants, soils, and oceans of interest to medicine, agriculture and conservation. The modeling task is to distill this detail into a minimum set of assumptions relevant to conceptual clarity, not to empirical completeness. For example, two versions of model are introduced. In one, almost all of the microbiome is transmitted vertically with but a trickle of horizontal mixing; in the other 
all the microbiome is transmitted horizontally without any vertical transmission. In reality of course, both modes occur simultaneously. That empirical truth is not relevant here, however. If holobiont selection is effective with near total vertical transmission and also with total horizontal transmission, then there is little reason to doubt it will also be effective if both modes of transmission occur simultaneously. It is sufficient for conceptual clarity to show that holobiont selection works in the two extreme cases, and to leave formulation of a model where some fraction of the transmission is vertical and the remaining fraction horizontal to the future should the need for such an elaborate model arise. I hope the reader will appreciate that simple models such as these are always vulnerable to attack for leaving out some cherished detail, and will grant me license to concentrate on assumptions that seem most relevant to bringing conceptual clarity to the holobiont vs. coevolutionary selection dispute. The models in this article are minimum models for concepts, not minimum models for empirical systems.

\section{The Hologenome}

Because the hologenome consists of the genes from all the microbes in the microbiome plus the host's nuclear genes, the mere description of the hologenome might seem daunting. The approach introduced here is to represent a hologenome as a hierarchy.

Figure 1 diagrams two instances of a conceptual holobiont, $A$ and $B$. A representative host is depicted as a rectangle with rounded corners. A host's genome contains nuclear genes depicted in the gray gear box. Holobiont- $A$ has allele $H_{1}$ and Holobiont- $B$ has $H_{2}$ at a locus of interest in its host's nucleus. Each holobiont has two niches for microbes depicted as green or brown that can represent different physical locations within the host or different functional roles. Two taxa of greens, circle and star, and two taxa of browns, rectangle and diamond, are available for each niche. The abundance of these taxa in each niche can vary among holobionts-Holobiont- $A$ has three circles and Holobiont $-B$ two stars. The allelic composition can vary among microbes- the circles have one $C_{1}$ allele and two $C_{2}$ alleles in Holobiont- $A$.

The hologenotype of an individual holobiont may be represented as a hierarchical data structure (list of lists) as depicted in Figure 1. The outer list indicates the number of genomes in the holobiont. (The host nucleus is counted as a taxon containing one genome.) Nested within this are lists for the abundance and identity of each genome. And nested within this are lists for the number and identity of each allele.

In the hologenotype description the number of microbes is normalized to a standard host cell. For example, if Holobiont- $A$ as a whole is comprised of 1000 host cells, 3000 green circle microbes, and 2000 brown rectangle microbes, then its hologenotype description, $\mathbf{H}_{A}$, records 1 host nucleus, 3 green circles, and 2 brown rectangles, as shown in Figure 1 .

A model of holobiont evolution predicts the trajectory through time of the hologenotype numbers for a holobiont population.

Burke, Steinberg, Rusch, Kjellberg, and Thomas (2011) have suggested that a bacterial community should be described in terms of bacterial functions rather than bacterial species. The hologenotype description in Figure 1 includes this possibility with its use of niches. A niche can be a region of the host, say gut vs. mouth, etc. But a niche can also be a functional role that can be played by any of several bacterial species. This later interpretation accords with common usage in ecology where community structure is also described in terms of niches-in island lizards in the Caribbean for example, distinct functional niches refer to species who feed in grass fields and on bushes, on tree trunks near the ground, higher on tree trunks, in the central canopy of trees, and on canopy twigs. These characteristic niches are populated by different species on different islands and in different habitats, yielding a recurring community structure

๑ OPEN ACCESS - PTPBIO.ORG 


\section{Hologenotypes with Two Host Niches and Four Microbial Taxa}

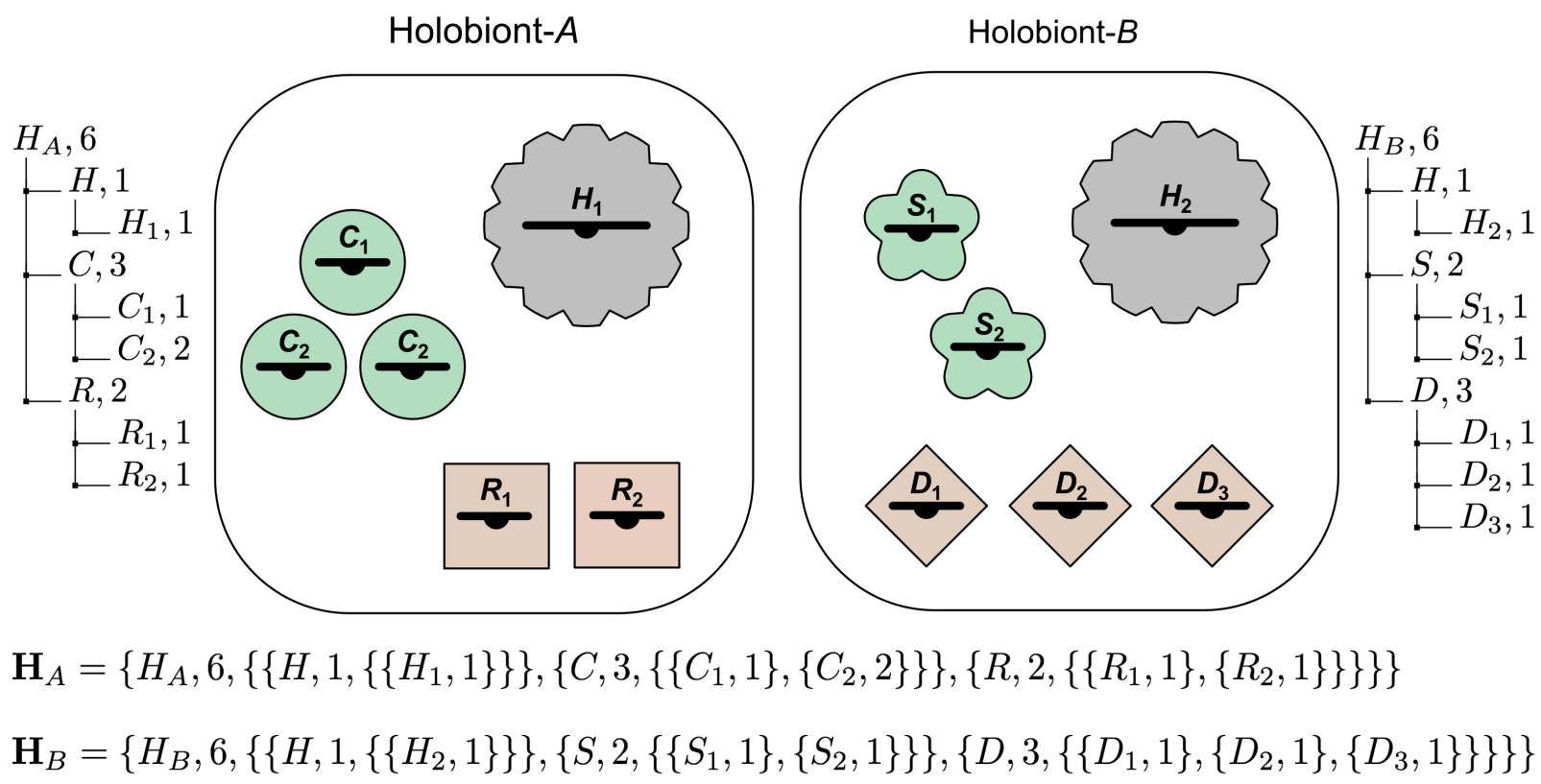

Figure 1: Examples of hologenotypes from two hosts assuming two microbe niches (green and brown) and two possible microbe taxa per niche (circles and stars, rectangles and diamonds) together with allelic genetic variation within the microbe taxa. The hologenotype may be represented as a list of lists, as illustrated at bottom of figure. The right and left sides of the figure illustrate the genetic hierarchy for the hologenotypes. $\mathbf{H}_{A}$, for example, reads as consisting of six individual genomes, one genome being the host nucleus containing one $H_{1}$ allele, three genomes from circle microbes containing among them one $C_{1}$ allele and two $C_{2}$ alleles, and two genomes from rectangle microbes containing among them one $R_{1}$ allele and one $R_{2}$ allele.

from place to place even though the species identities in those places vary (cf. Roughgarden 1995; Losos 2011). The suggestion that bacterial communities should be described in terms of bacterial functions rather than species accords with how community ecology has long described community structure in terms of niches.

Similarly, Doolittle and Booth (2015) and Doolittle and Inkpen (2018) have argued that selection pertaining to microbiomes should focus on the biochemical functions of bacteria rather than on their specific taxa. They write that biochemical functions may be conserved across hosts and through time whereas the taxa preforming those functions are variable. They argue that the functions themselves rather than the taxa should be considered as the objects of selection. This perspective is also accommodated with the type of hologenotype description illustrated in Figure 1. A hologenotype with, say, no green taxa could be matched against a hologenotype with no brown taxa. Holobiont selection in a holobiont population with these two hologenotypes would yield a result about the evolution of the niches themselves. Moreover, multiple functionally equivalent taxa could populate a niche, facilitating an investigation of within-niche species diversity based on within-niche neutral species.

Gene duplication is also covered by the hologenotype description of Figure 1. One can represent a duplicated nuclear gene with say, two copies of the $H_{1}$ allele in the the gray gear box 
of one hologenotype matched against one copy of the $H_{1}$ allele in the gray gear box of another hologenotype. Holobiont selection in a population with these two hologenotypes would reveal the evolutionary outcome of nuclear gene duplication.

Moreover, microbe abundance is in fact the equivalent of gene duplication-duplication of microbe genes rather than host nuclear genes. A hologenotype with say, two $C_{1}$ circle microbes could be matched against another hologenotype with say, three $C_{1}$ circle microbes. Holobiont selection with these two hologenotypes amounts to selection for the microbe's allele copy number. Alternatively, this selection could be viewed as selection for microbe abundance. Indeed, the role of the microbes can be looked at from either of two sides, as though an Escher painting. From the hologenome standpoint, the microbes are no more than encapsulated genes whose abundance represents a gene copy number. From a microbiology standpoint, the microbes are tiny living entities whose abundance represents the community structure of a microbiome. From the hologenome standpoint, holobiont selection is affecting gene copy number whereas from the microbiology standpoint, holobiont selection is affecting the microbiome community structure.

Thus, the hologenotype description of Figure 1 is very general and supports the future modeling of many evolutionary scenarios should the need arise. The goal in this paper is to compare the evolutionary outcomes of vertical and horizontal transmission. For this purpose it is sufficient here to analyze the special case of hologenotypic variation solely in microbe number, assuming one host niche, one microbial taxon, and no allelic variation in host or microbe genomes. The microbe may be either a pathogen or a mutualist.

First, the case of vertical transmission is developed, followed by the case of horizontal transmission.

\section{Vertical Transmission}

Three processes occur as sequential stages within each host generation, as shown in Figures 2 and 3 (Roughgarden 2017; Roughgarden et al. 2018). The host's generation time is the macro time step. The macro time step begins in the upper right of the figures, i.e., right after the holobiont selection stage and before the mixing stage. Within each macro time step, micro time steps reflect the kinetics of microbe exchange and microbe generation time. The text offers a verbal description of the model while the equations are found in the mathematical appendix.

\subsection{Model}

\subsubsection{Microbial Mixing}

Within a macro time step, the first stage is microbial mixing, as diagrammed at the top of Figures 2 and 3 .

Each microbe has a small probability, $m$, of exiting its holobiont and entering a temporary "transfer pool." Then the total transfer pool is divided equally across the holobionts. Each holobiont receives the same number of transfers. Hence, holobionts that had few microbes to begin with receive a net increase in microbe number and holobionts with many microbes to begin with incur a net decrease.

A small $m$ is consistent with limited microbe interchange across hosts. The holobiont population needs limited horizontal mixing even though parents primarily transmit microbes vertically to their offspring. Otherwise, if $m$ is 0 , then holobiont selection reduces to clone selection on the hosts whereby the host clone with lowest number of parasites or the largest number of mutualists becomes fixed. Furthermore, if $m$ is 0 , then empty hosts cannot be colonized. 


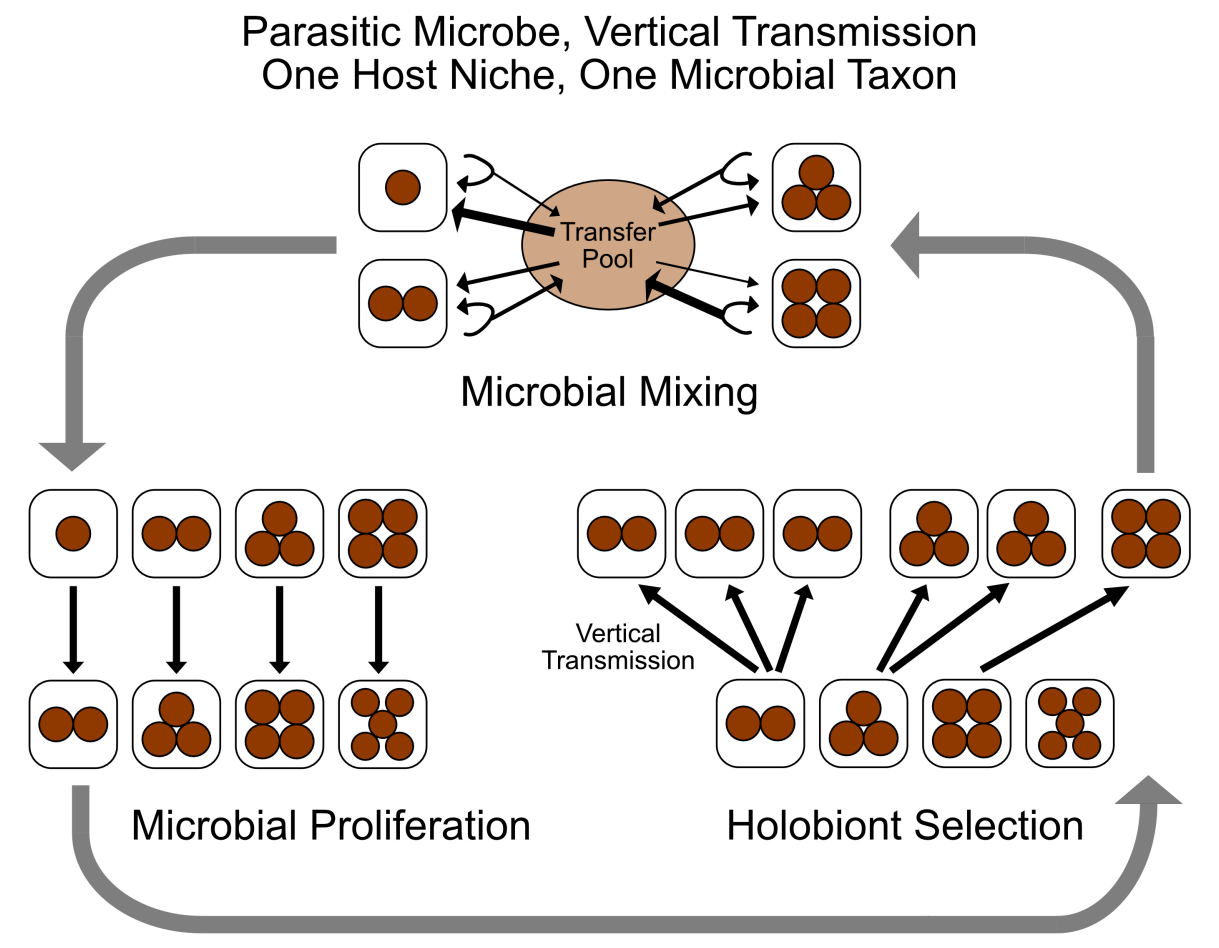

Figure 2: Sequence of processes with vertical transmission of parasitic microbiome.

\subsubsection{Microbial Proliferation}

After microbial mixing, the next stage is for the microbes to proliferate within the holobionts, as diagrammed on the left side of Figures 2 and 3 .

The microbes within each holobiont increase according to a density-dependent ecological population model. A single microbe strain obeys the logistic equation and multiple competing strains follow the Lotka-Volterra competition equations.

\subsubsection{Holobiont Selection}

Following microbial proliferation in the holobionts, the next stage is for the holobionts to be processed by holobiont selection, as diagrammed on the right side of Figures 2 and 3 .

Each holobiont reproduces as a whole. The number of progeny a holobiont produces depends on the number of microbes in it, which is its holophenotype. Parental holobionts transmit their microbes to their juveniles such that the number of microbes in each juvenile equals the number of microbes present in its parent. In the case of microbial parasites in Figure 2, the holobionts with the lowest number of microbes leave the largest number of progeny and conversely for microbial mutualists in Figure 3. Holobiont reproduction and survival are independent of the density of holobionts.

The macro time step ends after the holobiont selection stage, whereupon the holobiont population is ready for another macro time step that begins once again at the mixing stage. Iteration of the macro time step with these three stages generates a trajectory of holobiont abundance and hologenotype frequencies through time.

The mathematical appendix includes instructions for how to download the model's computer program from the website GitHub. Also, the appendix includes a link to a short five-minute video on YouTube illustrating how to run the computer program. 


\section{Mutualistic Microbe, Vertical Transmission \\ One Host Niche, One Microbial Taxon}

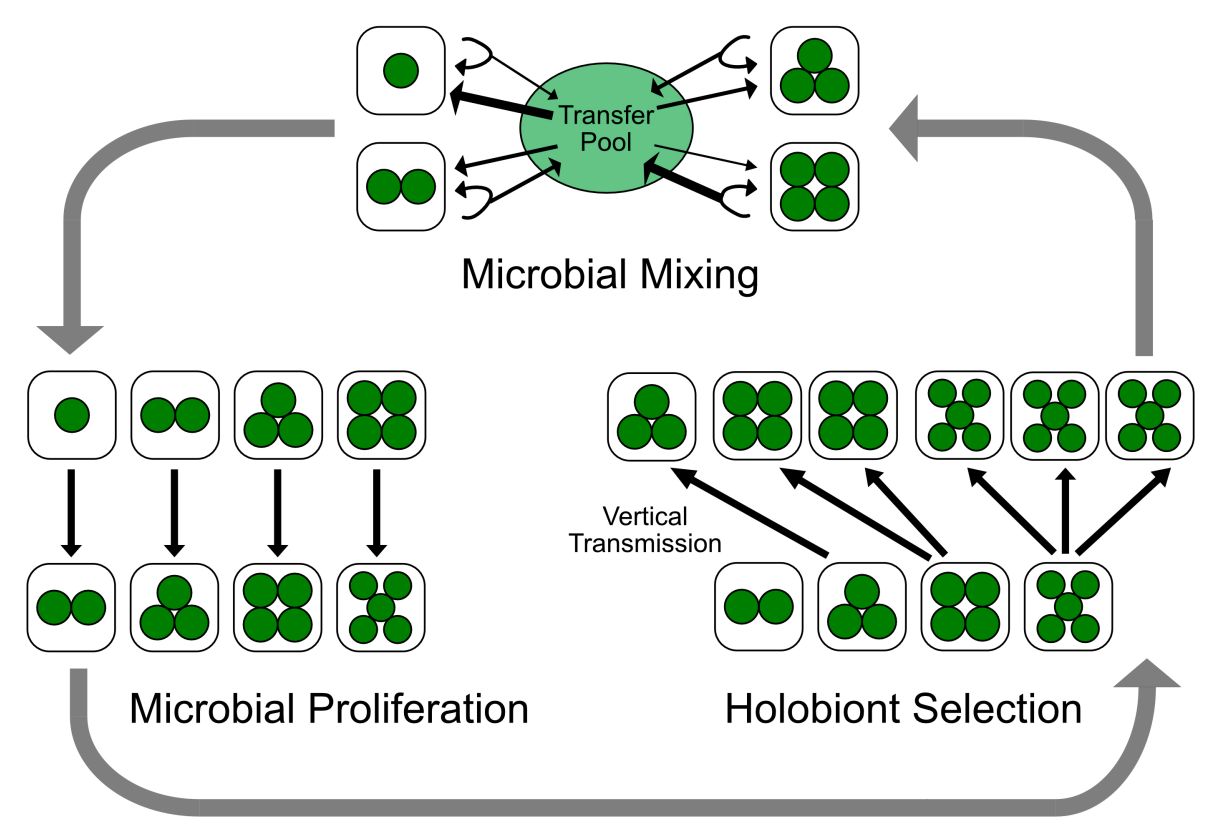

Figure 3: Sequence of processes with vertical transmission of mutualistic microbiome.

\subsection{Results}

Figures 4 and 5 show screen shots of the histogram through time of the hologenotype frequencies for host interactions with parasitic and mutualistic microbes. In both figures iteration of macro time steps leads from an initial uniform distribution to a stationary distribution of hologenotype frequencies at which holobiont selection balances microbial mixing.

In Figure 4 holobiont selection leads to a preponderance of holobionts with fewer parasites than initially. In Figure 5 holobiont selection leads to a preponderance of holobionts with more mutualists than initially. In the model's picture display (not shown), the holobionts with a parasitic microbiome change through time from $50 \%$ brown to almost no color and holobionts with a mutualistic microbiome change through time from $50 \%$ green to nearly solid green.

The stationary distribution of hologenotype frequencies partly depends on the initial condition. An historical accident affecting the initial condition may influence the outcome of holobiont selection in this scheme of microbial mixing.

Microbial mixing homogenizes the hologenome and retards the progress of holobiont selection, reinforcing an intuition that effective holobiont selection requires faithful vertical transmission of microbes with limited horizontal mixing.

For parasites, microbial proliferation acts in opposition to holobiont selection. By increasing the microbe's intrinsic rate of increase and/or the number of micro time steps the virulence of the parasite is increased. This increased virulence can overtake holobiont selection, leading to holobiont extinction.

These findings support comparable conclusions about the evolution of gut microbiota via host epithelial selection (Schluter and Foster 2012).

In reality, much of the microbiome does not colonize vertically. Thus, another variant of the model is needed. One possible modification to the model is to allow the mixing probability, $m$, to equal 1 , implying that every microbe leaves its host to enter the transfer pool only to be 


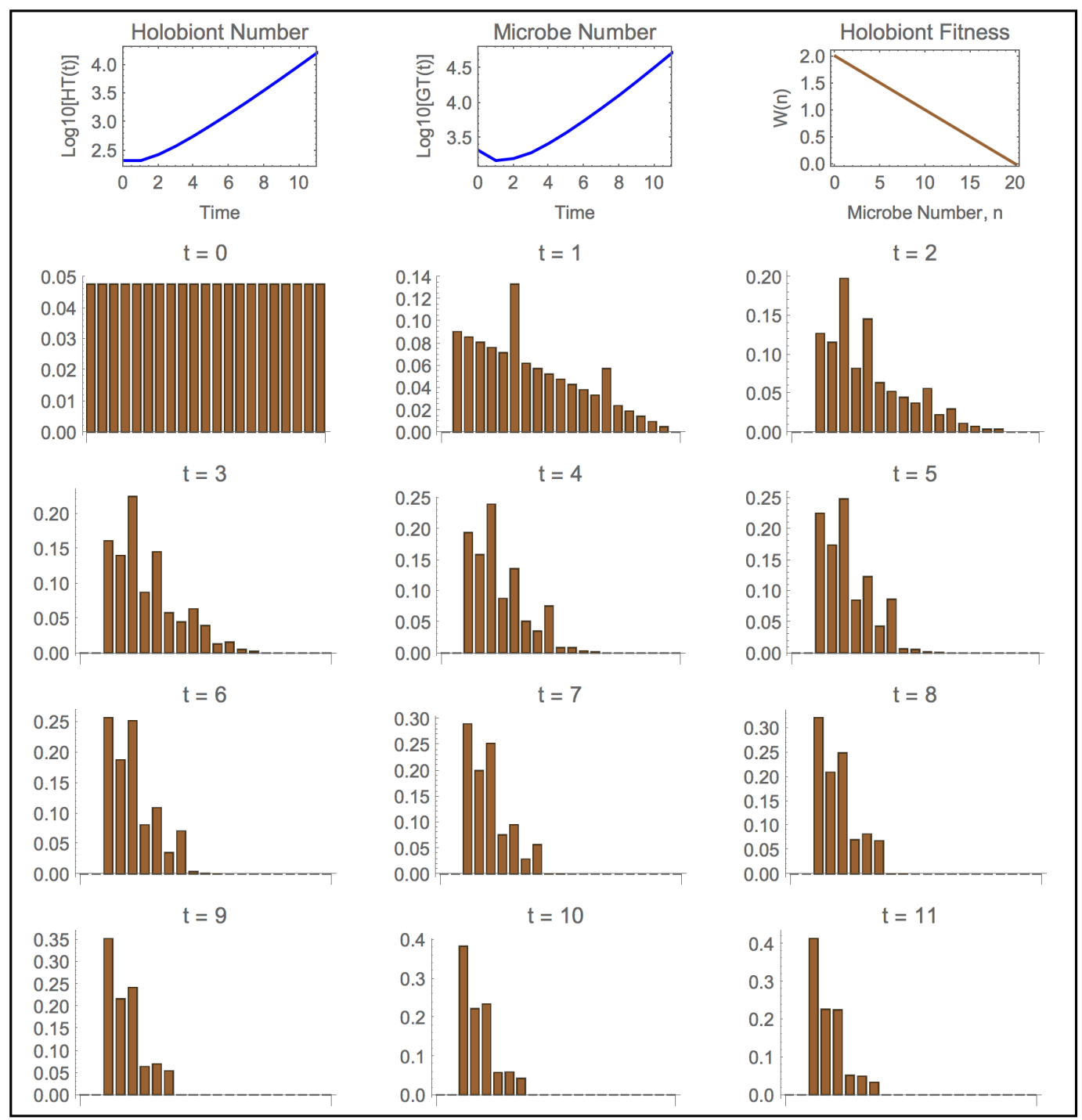

Figure 4: Results with vertical transmission of parasitic microbiome. Top left and middle graphs show $\log$ of total holobiont and microbe numbers through time and top right graph shows holobiont fitness as function of number of microbes in host. Histograms show distribution of hologenotype frequencies through time. The horizontal axis in the histograms is number of microbes per host and the vertical axis is fraction of holobiont population.

redistributed in equal measure back into now empty hosts. However, that would instantaneously stock all the hosts with identical microbiomes, denying holobiont selection any hologenotypic variation to operate on. Instead, sampling during microbe transmission can be introduced to the model. This sampling produces hologenotypic variation across holobionts and leads to the following formulation. 


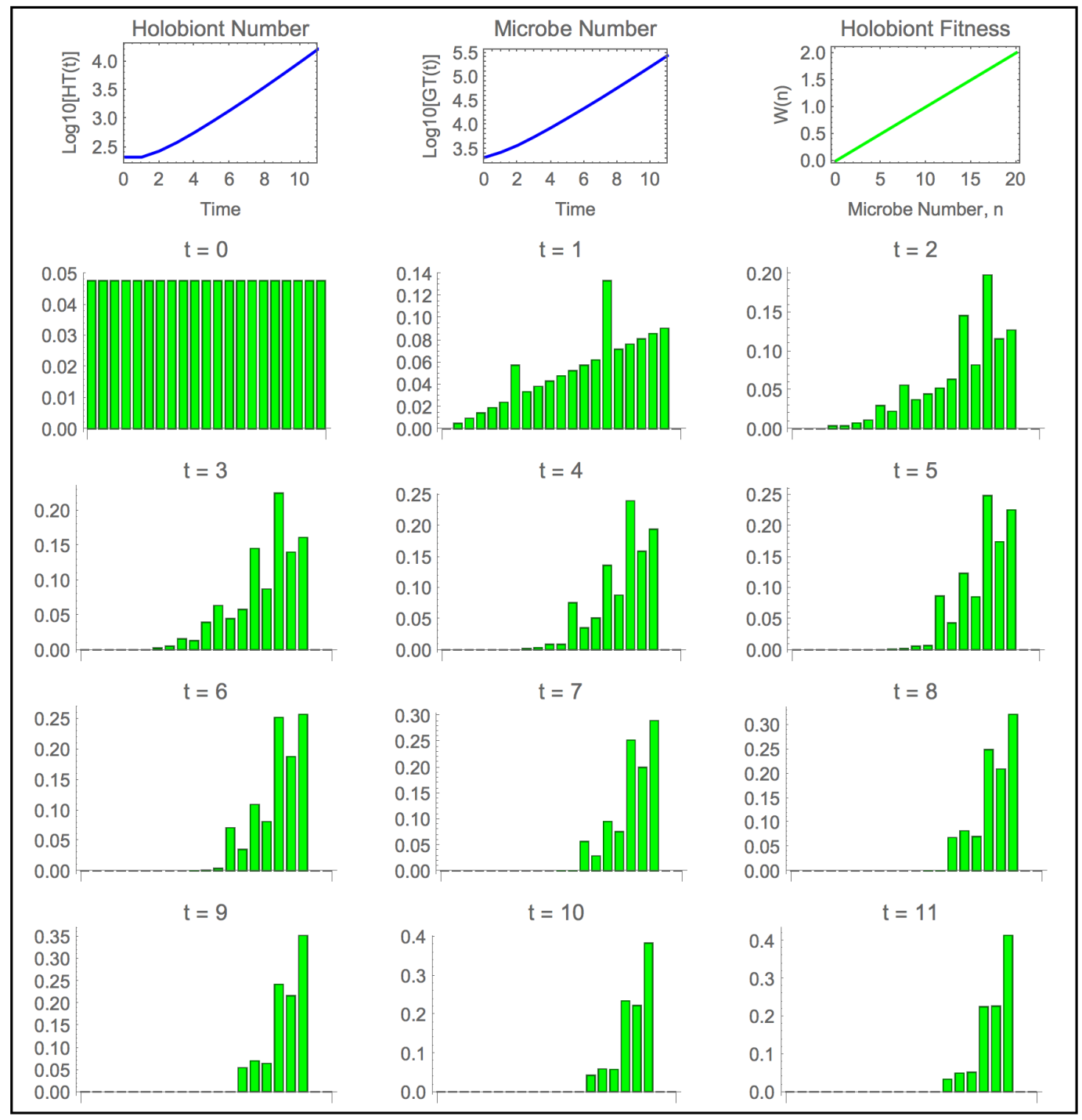

Figure 5: Results with vertical transmission of mutualistic microbiome.

\section{Horizontal Transmission}

\subsection{Model}

Three processes occur as sequential stages within each host generation, as shown in the top panels of Figures 6 and 7 . In detail:

\subsubsection{Microbial Transmission}

Each generation begins with a normalized Poisson distribution of microbes across holobionts based on a density parameter equal to the number of microbes in its source pool divided by the number of juvenile hosts in its source pool. 


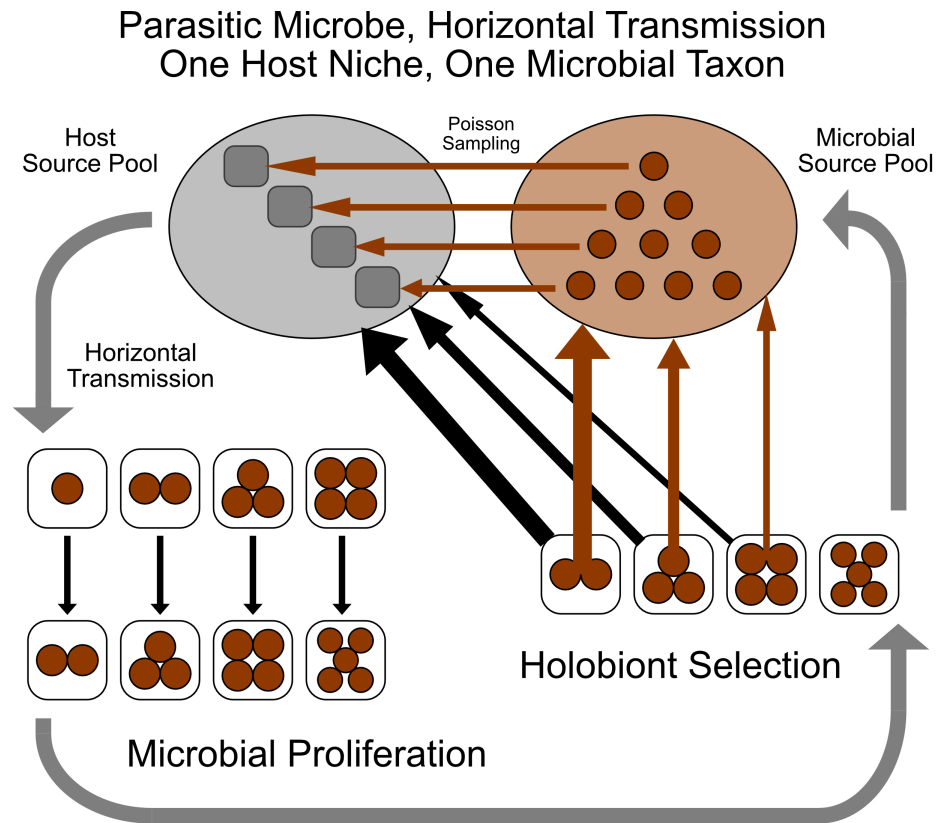

Figure 6: Sequence of processes with horizontal transmission of parasitic microbiome.

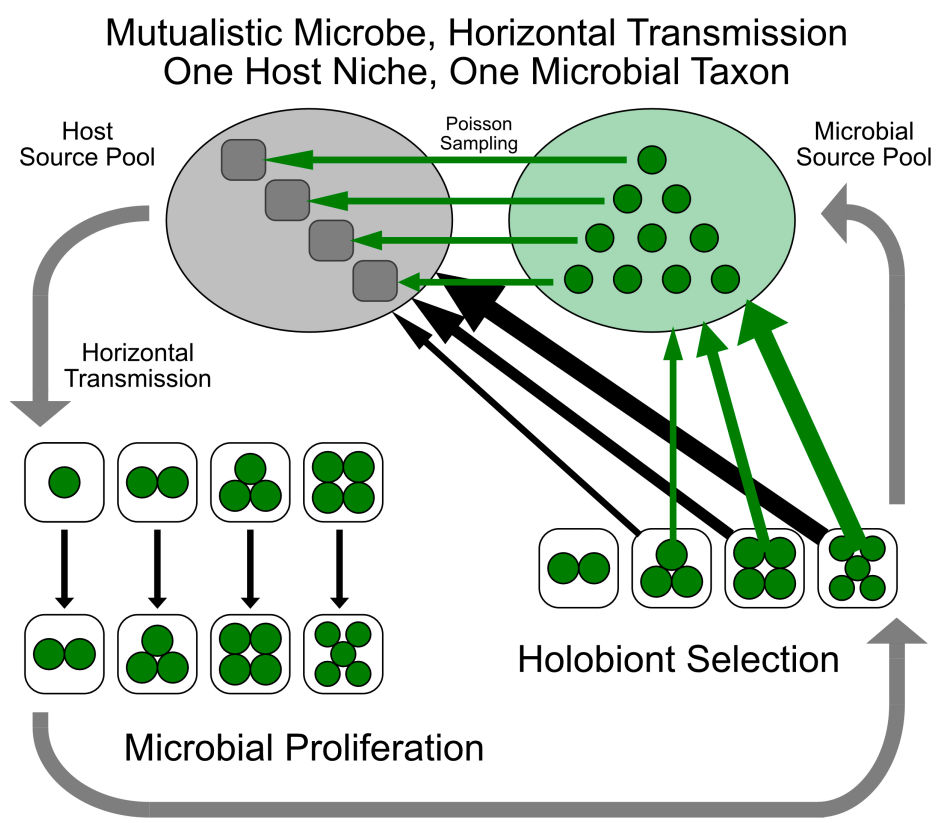

Figure 7: Sequence of processes with horizontal transmission of mutualistic microbiome.

\subsubsection{Microbial Proliferation}

The microbes within each holobiont increase according to density-dependent ecological population models, as before. 


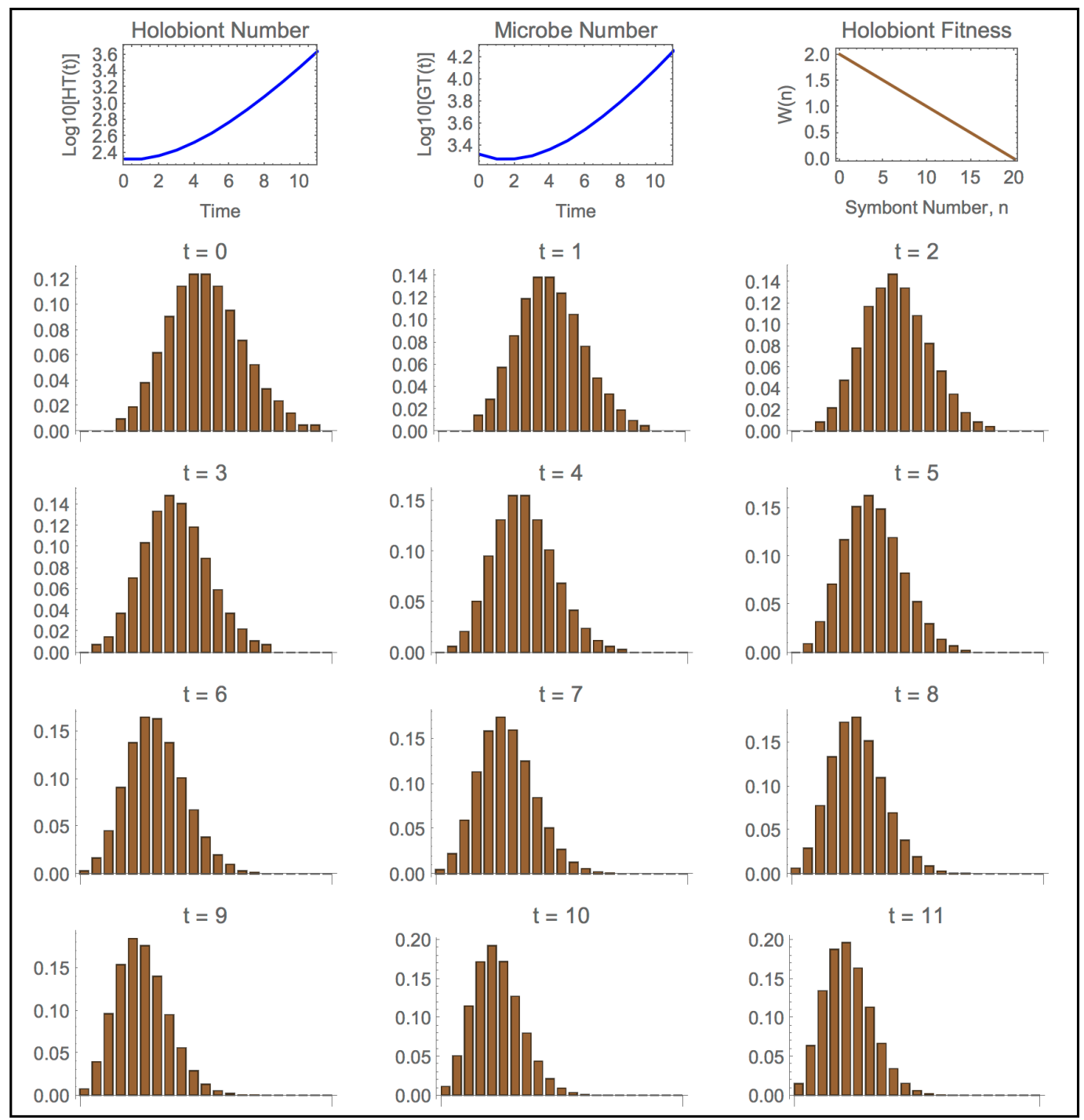

Figure 8: Results with horizontal transmission of parasitic microbiome.

\subsubsection{Holobiont Selection}

Each holobiont reproduces as a whole. The number of progeny a holobiont produces depends on the number of microbes in it. Upon reproduction, all the microbes produced from the holobionts enter a common microbe source pool, and all the juvenile hosts join a common host source pool. For microbial parasites, holobionts with the lowest number of microbes leave the largest number of progeny, and conversely for the microbial mutualists. Holobiont reproduction and survival is independent of the density of holobionts.

\subsection{Results}

Figures 8 and 9 show screen shots of the histogram through time of the hologenotype frequencies where the microbes are parasitic or mutualistic. Overall, the results are qualitatively similar in this model variant to those in the vertical transmission variant. Holobiont selection against parasites lowers average parasite microbe number in holobionts over generations relative to the 


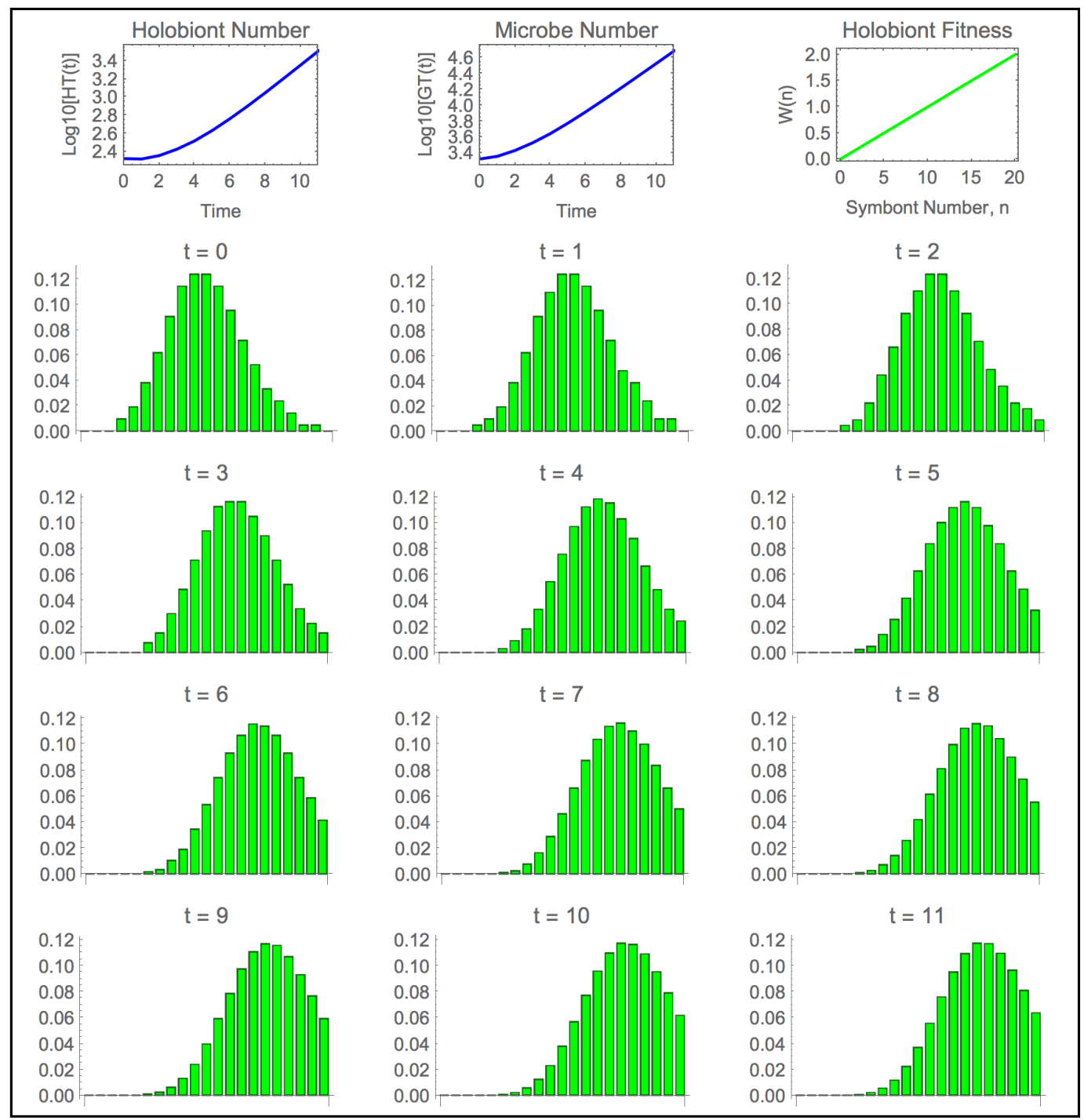

Figure 9: Results with horizontal transmission of mutualistic microbiome.

initial condition, and holobiont selection for mutualists increases average microbe number in holobionts over generations relative to the initial condition.

With horizontal transmission, a stationary distribution is attained when the holobiont selection balances the variability caused by the Poisson sampling of the source pools during transmission. (Whether this stationary distribution depends on initial conditions has not been analyzed.) Here too the virulence of the parasites can overcome the holobiont selection if the parasite intrinsic rate of increase is high enough and/or the number of micro time steps large enough.

\section{Discussion}

The model here shows that holobiont selection causes the evolution of hosts with a reduced number of parasites and an increased number of mutualists, assuming either vertical or horizontal microbiome transmission. This outcome is to be expected given that reducing parasite load and increasing mutualists are adaptive. But how this expected outcome is attained is what makes 
holobiont selection unique. In fact, three types of evolutionary processes can be distinguished that can influence microbe abundance, one of which is holobiont selection.

First, parasite abundance may be lowered and mutualist abundance increased via host control. If the host has a locus in its nuclear genome which produces an antibiotic that kills parasitic microbes, then ordinary natural selection will fix alleles at that locus resulting in a reduced parasite load. Similarly, if the host has a locus that produces a chemical facilitating mutualistic microbes, then ordinary natural selection will fix alleles at that locus, resulting in an increase of mutualists. A model in this framework has one equation-for changes in allele frequencies at a locus of interest in the host. This equation can be represented symbolically as a mapping from $p(t) \rightarrow p(t+1)$ where $p(t)$ is an allele frequency at the locus of interest at time $t$. This evolutionary process does not acknowledge that the microbes are alive. In this process, parasitic microbes are no different than abiotic toxins, and mutualistic microbes no different than abiotic nutrients. Therefore, host control does not seem to be a process capable of explaining the joint dynamics and evolution of the microbiome and host.

Second, the microbiome/host relationship can be influenced via coevolutionary selection. In a typical coevolutionary setup, the fitnesses influencing the gene frequencies in, say, species-1 are functions of the abundances of both species- 1 and species-2, and conversely for the fitnesses in species-2 (cf. Roughgarden 1983; Brown and Vincent 1987; Dieckmann and Law 1996; Carmona, Fitzpatrick, and Johnson 2015). This setup leads to two coupled equations for changes in the gene pools of the two species which can be represented symbolically as a mapping from $\{p(t) \rightarrow p(t+1), q(t) \rightarrow q(t+1)\}$ where $p(t)$ and $q(t)$ are allele frequencies in species-1 and species-2 at time $t$, respectively. The gene pools of the microbes and host are not merged and remain separate. The two species are playing an evolutionary game against each other, and the coevolutionary equilibrium is a Nash competitive equilibrium. This setup could be used to model how virulent a parasite should be, taking into account the corresponding negative response by the host. Such coevolutionary selection would cause a net increase or decrease in microbe and/or host abundance depending on the feedbacks to their population dynamics. In a similar vein, mutualists who "cheat" by short-changing the benefits delivered to their host are thought to be repressed by host sanctions and other forms of retaliation (West et al. 2002; Frederickson 2013). A mutualism may also be stabilized with reciprocal rewards (Kiers, Duhamel, Beesetty, et al. 2011). In any case, the flavor of a coevolutionary account of mutualism is to emphasize a transactional "give and take" between antagonistic parties. This perspective has recently been extended to host/microbiome cooperation as well, where it is argued that "the host and each individual microbial strain are distinct entities with potentially divergent selective pressures" and further, that host control is needed to constrain the microbiome, to keep the microbiome on a "leash" (Foster et al. 2017, 43).

Third, the microbiome/host relationship can be influenced via holobiont selection, as has been demonstrated here. In holobiont selection, the gene pools of the microbes and host are merged into a single hologene pool, and selection acts on a holobiont as a unit. The model consists of one "equation"; that is, the program to compute the vector of hologenotype numbers at time, $t+1$, given the vector at time, $t$, is a single mapping that can be represented symbolically as $H(t) \rightarrow H(t+1)$. Thus, holobiont selection is a logical counterpart to ordinary natural selection, but based on hologenotypes rather than on traditional Mendelian genotypes.

Whether holobiont selection is more important to holobiont evolution than coevolutionary selection is an open empirical question. The paper here shows that holobiont selection is a theoretically plausible evolutionary process. Holobiont proponents are therefore entitled to conjecture that holobiont selection is more effective in causing the evolution of microbiome/host integration than coevolutionary selection is. 
Although holobiont selection leads to the same qualitative outcome with both vertical and horizontal transmission, the underlying evolutionary processes are different between these two modes of transmission.

In vertical transmission, microbes incorporate into a host lineage along with the host's nuclear genome. Microbial mixing loosens the association between microbes and the success of their holobiont's lineage, thereby reducing the impact of the holobiont selection. Mixing also homogenizes the hologenotypes across holobionts, erasing the hologenotypic variation that holobiont selection relies on to produce an evolutionary response.

In horizontal transmission, beneficial microbes from hosts that are successful because of having many mutualists flood the microbial source pool. Hence, the next generation's holobionts are assembled with an increase in microbe number because the ratio of microbes to hosts has increased. Conversely, the limited production of parasitic microbes from hosts that are successful because of having few parasites dilutes the microbial source pool. Hence, the next generation's holobionts are assembled with a decrease in number of microbes because the ratio of microbes to hosts has decreased. The Poisson sampling of the microbes colonizing hosts guarantees hologenotypic variation for the holobiont selection to operate on.

With vertical transmission, a holobiont's microbiome is inherited by lineal descent, albeit not as a Mendelian trait. In contrast, the process of microbiome assembly from the combined parental microbe production is dubbed here collective inheritance.

Collective inheritance might prove valuable to understanding the evolution of bacteria and other microbes that feature large amounts of horizontal gene transfer (Mao and Lu 2016). It might also aid understanding cultural inheritance wherein offspring inherit a sample of the pooled knowledge of the parental generation.

A distinction between lineal and collective inheritance appears in the literature on multilevel selection in connection with the concepts of multi-level selection 1 (MLS1) and multilevel selection 2 (MLS2) (Mayo and Gilinsky 1987; Damuth and Heisler 1988; Okasha 2005, 2006). Okasha $(2005,1017)$ writes:

The key issue is whether the particles or the collectives constitute the 'focal' level, i.e., the level of interest. Are we interested in the frequency of different types of particle in the overall population of particles (which just so happens to be subdivided into collectives), or are we interested in the frequency of different types of collective themselves? If the former, then the collectives are significant only in that they constitute an environment for the particles, and thus may affect the particles' fitnesses. We will judge evolution to have occurred when the overall frequency of different particle-types has changed. If the latter, then we are interested in the collectives in their own right, not simply as environments of the particles. We will judge evolution to have occurred when the frequency of different collective-types has changed. Damuth and Heisler (1988) refer to the first approach as multilevel selection 1 (MLS1), and the latter as multilevel selection 2 (MLS2).

Okasha $(2006,58)$ further writes:

In MLS2, collective fitness is defined as the number of offspring collectives. For this notion to apply, it is essential that the collectives reproduce in the ordinary sense, that is, they 'make more' collectives; otherwise, determining parent-offspring relations at the collective level will not exist. But in MLS1 this is inessential. The role of the collectives in MLS1 is to generate a population structure for the particles, which affects their fitnesses. For MLS1 to produce sustained evolutionary conse- 
quences, collectives must 'reappear' regularly down the generations, but there is no reason why the collectives themselves must stand in parent-offspring relations.

Roughly speaking, holobiont selection with horizontal transmission corresponds with MLS1 and holobiont selection with vertical transmission corresponds with MLS2. But, holobiont evolution is not really the same as what these quotes are describing. In MLS1 the focus is on the microbes, with the host being secondary. Indeed, the host, or "collective" of MLS1 need not even be an organism. The collective need only be a kind of location, like the recurring haystacks in a field within which mice, the "particles", can interact and realize their fitnesses in that context (Maynard Smith 1964). In contrast, with MLS2 the focus is on the host, with the microbes being secondary. The collectives give birth to more collectives depending on the particles in them while passing along their particles to their offspring collectives, so both the collective and their particles share the same lineal parent-offspring relation.

Now, in holobiont evolution, both host and microbes are equally important assuming either mode of microbiome transmission, and neither is primary or secondary, because both the host and microbiome together comprise the holobiont itself and both participate in holobiont selection. Still, the distinction between MLS1 and MLS2 does foreshadow the distinction between lineal and collective inheritance encountered in the theory of holobiont evolution. Thus, although similarities between holobiont selection and multi-level selection exist, it may be fair to say that holobiont selection theory is not an extension of multi-level selection theory and that holobiont selection should be taken as a distinct process in its own right, sui generis.

Both lineal and collective modes of inheritance, although not named as such, are included in a recent model of microbiome evolution featuring both host and microbial selection (Zeng et al. 2017). Further, the model here shares some similarity with literature on the evolution of organelles (Birky, Maruyama, and Fuerst 1983; Rand, Hane, and Fry 2004; Smith 2007).

In a recent multilevel selection model for host/microbiome evolution, van Vliet and Doebeli $(2019,5)$ concluded that "selection at the host level and in extension, at the holobiont level, could be of importance in nature but only under rather restrictive circumstances" including a requirement that "vertical transmission has to dominant over horizontal transmission." The van Vliet-Doebeli model is an extension of a previous model described as a "mathematical formulation of Type II group selection" (Simon, Fletcher, and Doebeli 2012, 1569). As such, the van Vliet-Doebeli model is comparable to the vertical-transmission version of the model in this paper-its conclusions are not relevant to the horizontal-transmission version.

I have two remarks about the van Vliet-Doebeli paper. First, the result that holobiont selection is effective with a vertically transmitted microbiome only if the vertical transmission is not diluted too much by horizontal mixing has already been shown in Roughgarden 2017 and Roughgarden et al. 2018, and is also mentioned in this paper when discussing the vertical-transmission case-but again, this concern does not pertain to microbial mixing in the horizontal-transmission case.

Second, van Vliet and Doebeli $(2019,1)$ refer to a preprint of this paper (Roughgarden 2019), dismissing it with the claim that "by excluding variation in microbiome composition, this model could not address the question of under what conditions selection at the holobiont level can maintain traits that are disfavored by selection at the microbe level." This assertion is neither fair nor correct. After all, this paper is about vertical vs. horizontal transmission-it did not intend to address the question of microbe/host tradeoffs. However, one could extend this paper's model to investigate a tradeoff between microbe and host fitnesses if one wanted to.

Previous investigators have noted that holobiont evolution is, in a sense, Lamarckian because holobiont selection affects acquired rather than inherited traits (Rosenberg, Sharon, and ZilberRosenberg 2009; Osmanovic et al. 2018). With horizontal transmission especially, a holobiont's 
microbial abundance is acquired from environmental source pools. Still, evolution via holobiont selection seems best viewed as Darwinian rather than Lamarckian, because holobiont selection is a selection process acting on holobionts as individuals. However, evolution via holobiont selection is not neo-Darwinian because neo-Darwinism is founded on Mendelian inheritance (Wright 1931).

The model here and its framework are extensible and scalable. Future theoretical research might investigate the comparative effectiveness of holobiont and coevolutionary selection in producing host/microbiome integration, how holobiont selection modulates microbiome community structure, and how species diversity is maintained in the microbiome.

\section{Conclusion}

The model here with its two variants demonstrates theoretically that holobiont selection is plausibly an important force in evolution, because holobiont selection produces evolutionary change in holobionts with both vertical and horizontal microbe host transmission. Holobiont evolution can rely on collective inheritance as well as lineal inheritance.

\section{Appendix A: Mathematical Details}

\section{A1 State Variable Defined}

The hologenotype numbers for a holobiont population through time are recorded in the matrix, $H$, where $H(t, n)$ is the number of holobionts at time $t$ containing $n$ microbes. The top row of $H$ records the initial condition of the holobiont population. The model computes the row at $t+1$ given the row at $t$; that is, after each macro time step, the model appends a row to $H$. The index, $t$, runs from 1 , which is the initial condition, to $t_{M}+1$ where $t_{M}$ is the number of macro time steps in the iteration. The index, $n$, runs from 0 to $K$, where $K$ is the maximum number of microbes in a host. At the conclusion of $t_{M}$ macro time steps, $H$ has become a matrix with dimensions, $\left(t_{M}+1\right) \times(K+1)$.

The total number of holobionts through time is recorded in the vector

$$
H_{T}(t)=\sum_{n=0}^{K} H(t, n)
$$

and the total number of microbes through time is recorded in the vector

$$
G_{T}(t)=\sum_{n=0}^{K} n H(t, n) .
$$

\section{A2 Model Equations - Vertical Transmission}

Here are the three stages within a macro time step for the vertical-transmission variant of the model.

\section{A2.1 Horizontal Microbe Transfer across Holobionts}

The total number number of microbes that enter the transfer pool is $m G_{T}(t)$, where $m$ is the probability a microbe leaves its host. Regardless of the host they reside in, all microbes have the same probability of entering the transfer pool. Therefore, the number of microbes who enter the 
transfer pool is simply $m$ times the total number of microbes summed over all holobionts. The transfer pool is then distributed back equally across the holobiont population. The number of transfers received back from the pool per holobiont is then $m G_{T}(t) / H_{T}(t)$, provided $H_{T}(t)>$ 0 . Every holobiont receives this number.

Putting the arrivals and departures together yields a formula for the new number of microbes, $n^{\prime}(t, n)$ in a holobiont that formerly contained $n$ microbes at time, $t$.

$$
n^{\prime}(t, n)=(1-m) n+m G_{T}(t) / H_{T}(t)
$$

This formula says the new number of microbes in the holobiont equals the number there previously minus the number that departed plus the number that arrived.

The $n^{\prime}(n)$ must be an integer. To avoid considering fractional microbes, Equation (3) is rewritten as

$$
n^{\prime}(t, n)=\left\lfloor(1-m) n+m G_{T}(t) / H_{T}(t)\right\rceil,
$$

where the symbol $\lfloor x\rceil$ indicates the nearest integer to $x$.

The number of holobionts with $n$ microbes after the horizontal transfer, $H^{\prime}(t, n)$, then is found by accumulating over $i$ the holobionts whose microbe composition has changed from $i$ to $n^{\prime}$.

$$
H^{\prime}(t, n)=\sum_{i=1}^{K} H(t, i)
$$

To see how this equation works, take a particular holobiont composition after horizontal transfer. Ask, for example, how many holobionts have exactly three microbes in them after the horizontal transfer has completed? $H^{\prime}(t, 3)$ is found by summing over $H$ before the transfer started and using only the terms for the holobionts who wound up with 3 microbes after the transfer was over. If the summation is indexed with $i$, then the terms to include in the summation are those for which $3=n^{\prime}(i)$. These are the terms for holobionts who started with $i$ microbes and wound up with 3 microbes. The same logic applies to $H^{\prime}(t, n)$ for any $n$.

\section{A2.2 Microbe Proliferation within Holobionts}

The next step is for the microbes to proliferate within their holobionts. If a holobiont starts with $n$ microbes, then after one micro time step, the number of microbes in it is $F(n)$, where $F(n)$ is a demographic model for population growth.

The widely used logistic equation predicts sigmoid population growth with the population size leveling off at $K$, the carrying capacity. The speed of population growth initially, is $r$, the intrinsic rate of increase. Here, the model is written using a general $F(n)$ to indicate that any suitable demographic model may be used, not necessarily the logistic equation.

Let $t_{m}$ be the number of micro time steps per macro time step. After $t_{m}$ steps, the microbe population will have grown in size to

$$
n^{\prime \prime}(n)=F^{t_{m}}(n)
$$

where $F^{t_{m}}(n)$ is the $t_{m}^{\text {th }}$ iterate of $F(n)$. To avoid fractional microbes, the nearest integer is taken, yielding

$$
n^{\prime \prime}(n)=\left\lfloor F^{t_{m}}(n)\right\rceil \text {. }
$$

The number of holobionts with $n$ microbes after microbe proliferation, $H^{\prime \prime}(n)$, then is found using the same logic as in the preceding stage, by accumulating over $i$ the holobionts whose 
microbe composition has changed from $i$ to $n^{\prime \prime}$.

$$
H^{\prime \prime}(t, n)=\sum_{i=1 \mid}^{K} H^{\prime}(t, i)
$$

The microbe proliferation takes place in the holobiont population after the horizontal transfer stage. Hence, $H^{\prime}(t, n)$ appears in Equation (8).

\section{A2.3 Holobiont Selection}

Here the number of microbes within a holobiont does not change, but the number of holobionts changes. The fitness of a holobiont with $n$ microbes, defined as the number of progeny that the holobiont produces, is $W(n)$. Therefore, the number of holobionts with $n$ microbes after reproduction is simply

$$
H(t+1, n)=W(n) H^{\prime \prime}(t, n) .
$$

The holobiont selection takes place after microbe proliferation stage. Hence, $H^{\prime \prime}(t, n)$ appears in Equation (9). Fractional holobionts are not meaningful, so the preceding equation is modified to indicate that the nearest integer is intended:

$$
H(t+1, n)=\left\lfloor W(n) H^{\prime \prime}(t, n)\right\rceil .
$$

Overall, Equation (10) predicts the hologenotype numbers at time $t+1$ based on what it was at time $t$.

\section{A3 Model Equations-Horizontal transmission}

Here are the three stages within a macro time step for the horizontal-transmission variant of the model.

\section{A3.1 Horizontal Transmission from Source Pools}

The macro time step begins with values for the size of the host pool, $H_{T}(t)$, and the microbe pool, $G_{T}(t)$. Then the Poisson density parameter indicating the average number of microbes per host is

$$
\mu(t)=\frac{G_{T}(t)}{H_{T}(t)} .
$$

Next, the hologenotype numbers are found from a Poisson density function, $P_{\mu}(n)$, normalized on the interval, $[0, K]$, multiplied by the number of hosts at time $t$,

$$
H^{\prime}(t, n)=\left\lfloor\frac{P_{\mu(t)}(n)}{\sum_{n=0}^{K} P_{\mu(t)}(n)} H_{T}(t)\right\rceil,
$$

where $P_{\mu(t)}(n)=\frac{e^{-\mu(t)} \mu(t)^{n}}{n !}$ for $n \geq 0$. The numerator of the fraction in the equation above is the probability that an offspring of the host is colonized by $n$ microbes given that the average number of microbes per host is $\mu(t)$ according to a Poisson probability distribution. However, the number of microbes in a host cannot exceed the host carrying capacity for microbes, $K$. So, the sum of probabilities of $n$ from 0 to $K$ is taken as the denominator in the fraction above. Thus, the overall probability of an offspring of the host being colonized by $n$ microbes where $n$ is between 0 and $K$ is the fraction in the formula above. The sum of this fraction from 0 to $K$ equals 1 , as it should. This fraction then multiplies the number of hosts waiting to be colonized, $H_{T}(t)$, to yield the number of host offspring with $n$ microbes at time $t$. 


\section{A3.2 Microbe Proliferation within Holobionts}

As before, the next step is microbe proliferation within their holobionts. $F(n)$ is a demographic model for population growth, such as the logistic equation.

After $t_{m}$ steps, the microbe population grows in size to

$$
n^{\prime \prime}(n)=\left\lfloor F^{t_{m}}(n)\right\rceil,
$$

where $F^{t_{m}}(n)$ is the $t_{m}^{\text {th }}$ iterate of $F(n)$.

The number of holobionts with $n$ microbes after microbe proliferation, $H^{\prime \prime}(n)$, is found by accumulating over $i$ the holobionts whose microbe composition has changed from $i$ to $n^{\prime \prime}$.

$$
H^{\prime \prime}(t, n)=\sum_{i=1 \mid n=n^{\prime \prime}(i)}^{K} H^{\prime}(t, i)
$$

\section{A3.3 Holobiont Selection}

The fitness of a holobiont with $n$ microbes, defined as the number of progeny that the holobiont produces, is $W(n)$. Therefore, the number of holobionts with $n$ microbes after reproduction is simply

$$
H(t+1, n)=\left\lfloor W(n) H^{\prime \prime}(t, n)\right\rceil .
$$

Prior to the next macro time step, all the microbes leave their holobionts and join a microbe source pool and the now-empty holobionts join a host source pool. Microbe retransmission and holobiont reassembly commences at the start of the next macro time step. The number of hosts and microbes in their source pools are, respectively,

$$
H_{T}(t+1)=\sum_{n=0}^{K} H(t+1, n)
$$

and

$$
G_{T}(t+1)=\sum_{n=0}^{K} n H(t+1, n) .
$$

A new value of Poisson density parameter describing the average number of microbes per holobiont, $\mu(t+1)$, is then computed for use at the beginning of the next macro time step.

\section{A4 Download Program}

Short programs written in Mathematica are available for download. The programs are supplied as both Mathematica Notebooks with an .nb extension and as Computable Document Format (CDF) files with an .cdf extension.

To use the .nb files one must have the Mathematica app which is quite expensive, although available in many universities. The .nb files allow the user not only to run the programs and also to adapt and change them by modifying the Mathematica code.

Using the CDF files requires only a free CDF player that may be downloaded from Wolfram. The player is analogous in principle to a PDF reader, only here CDF files rather than PDF files are displayed. The CDF files are fully interactive and one can change the model parameters with sliders. The only limitation to using CDF files rather than the notebook files is that one cannot modify the code of a CDF file.

\footnotetext{
${ }^{1}$ https://github.com/JoanKauai/Holobiont-Dynamics-Evolution

${ }^{2} \mathrm{https} / / / \mathrm{www} . w o l f r a m . c o m / c d f-p l a y e r /$
} 
If one is uncertain about whether to download the CDF player, one can use the CDF files directly online with a web browser. ${ }^{3}$ The CDF files run much faster when using the CDF player on a local computer, but clicking on these links provides a preview of how the programs work prior to deciding whether to download the player. A short five-minute video demonstrating how the software works is also available. ${ }^{4}$

\section{Acknowledgments}

I am deeply grateful to Erol Akçay, Scott Gilbert, Priya Iyer, Ehud Lamm, Eugene Rosenberg, Jeremy Van Cleve, Vignesh Venkateswaran, and Ilana Zilber-Rosenberg for valuable comments on the manuscript. I thank two anonymous reviewers of this manuscript and an anonymous reviewer of a previous version for helpful comments.

\section{Literature cited}

Babcock, R. C., G. D. Bull, P. L. Harrison, A. J. Heyward, J. K. Oliver, C. C. Wallace and B. L. Willis. 1986. "Synchronous Spawnings of 105 Scleractinian Coral Species on the Great Barrier Reef.” Marine Biology 90: 379-394.

Birky, C. W. Jr., T. Maruyama, and P. Fuerst. 1983. "An Approach to Population and Evolutionary Genetic Theory for Genes in Mitochondria and Chloroplasts, and Some Results.” Genetics 103: 513-527.

Björk J., C. Díez-Vives, C. Astudillo-García, E. A. Archie, and J. Montoya. 2019. "Vertical Transmission of Sponge Microbiota is Inconsistent and Unfaithful." Nature Ecology and Evolution. doi: 10.1038/s41559-019-0935-x.

Booth, A. 2014. "Symbiosis, Selection, and Individuality." Biol. Philos. 29: 657-673.

Bordenstein, S. R., and K. R. Theis. 2015. "Host Biology in Light of the Microbiome: Ten Principles of Holobionts and Hologenomes.” PLoS Biol. 13(8):e1002226.

Brown, J. S., and T. L. Vincent. 1987. "Coevolution as an Evolutionary Game.” Evolution 41: 66-79.

Burke, C., P. Steinberg, D. Rusch, S. Kjellberg, and T. Thomas. 2011. "Bacterial Community Assembly Based on Functional Genes rather than Species.” Proc. Natl. Acad. Sci. (USA) 108(34): 14288-14293.

Carmona, D., C. R. Fitzpatrick, and M. T. Johnson. 2015. "Fifty years of Co-evolution and Beyond: Integrating Co-evolution from Molecules to Species.” Mol. Ecol. 24: 5315-5329.

Damuth, J., and L. Heisler. 1988, Alternative Formulations of Multilevel Selection.” Biology and Philosophy 3: 407-430.

Dieckmann, U., and R. Law. 1996. "The Dynamical Theory of Coevolution: A Derivation from Stochastic Ecological Processes.” J. Math. Biol. 34: 579-612.

Doolittle, W. F., and A. Booth. 2017. "It's the Song, Not the Singer: An Exploration of Holobiosis and Evolutionary Theory." Biol. Philos. 32(1): 5-24.

Doolittle, W. F., and S. A. Inkpen. 2018. "Processes and Patterns of Interaction as Units of Selection: An Introduction to ITSNTS thinking." Proc. Nat. Acad. Sci. (USA) 115: 406-414.

\footnotetext{
${ }^{3}$ The vertical transmission version of the model is found at: https://www.wolframcloud.com/objects/joan.roughgarden/Published/HolobiontDynamicsVertical-05.cdf and the horizontal version at:

https://www.wolframcloud.com/objects/joan.roughgarden/Published/HolobiontDynamicsHorizontal-05.cdf. ${ }^{4}$ https://youtu.be/19w7ZDkRe18
} 
Douglas, A. E., and J. H. Werren. 2016. "Holes in the Hologenome: Why Host-Microbe Symbioses Are Not Holobionts." MBio 7(2):e02099-15. doi:10.1128/mBio.02099-15.

Dubilier, N., M. McFall-Ngai, and L. Zhao. 2015. "Microbiology: Create a Global Microbiome Effort." Nature. 526(7575): 631-4.

Foster, K. R., J. Schluter, K. Coyte, and S. Rakoff-Nahoum. 2017. "The Evolution of the Host Microbiome as an Ecosystem on a Leash." Nature 548: 43-51.

Fraune, S., and T. C. G. Bosch. 2010. "Why Bacteria Matter in Animal Development and Evolution." Bioessays 32: 571-580.

Gilbert, S. F., J. Sapp, and A. I. Tauber. 2012. "A Symbiotic View of Life: We Have Never Been Individuals." Q. Rev. Biol. 87: 325-341.

Gilbert, S. F., E. Rosenberg, and I. Zilber-Rosenberg. 2017. "The Holobiont with its Hologenome Is a Level of Selection in Evolution," In Landscapes of Collectivity in the Life Sciences, edited by S. B. Gissis, E. Lamm, and A. Shavit, 305-324. London: MIT Press.

Guerrero, R., L. Margulis, and M. Berlanga. 2013. "Symbiogenesis: the Holobiont as a Unit of Evolution.” International Microbiology 16: 133-143.

Hester, E. R., K. L. Barott, J. Nulton, M. Vermeij, and F. Rohwer. 2015. "Stable and Sporadic Symbiotic Communities of Coral and Algal Holobionts.” ISME J. 1-15.

Hirose, M., and M. Hidaka. 2006. "Early Development of Zooxanthella-containing Eggs of the Corals, Porites cylindirica and Montipora digitata: The Endodermal Localization of Zooxanthellae." Zoological Science 23: 873-881.

Hurst, G. D. D. 2017. “Extended Genomes: Symbiosis and Evolution.” Interface Focus 7: 20170001. doi:10.1098/rsfs.2017.0001.

Jefferson, R. 1994. “The Hologenome." Agriculture, Environment and the Developing World: A Future of $P C R$. Cold Spring Harbor, New York.

Jennings, E. C. 2019. "A Holobiont Characterization of Reproduction in a Live-bearing Cockroach, Diploptera punctata." PhD diss. Retrieved from https://etd.ohiolink.edu/.

Jennings, E. C., M. W. Korthauer, T. L. Hamilton, and J. B. Benoit. 2019. "Matrotrophic Viviparity Constrains Microbiome Acquisition During Gestation in a Live-bearing Cockroach, Diploptera punctata." Ecology and Evolution. 9 (18): 1-14. doi:10.1002/ece3.5580..

Kiers, E. T., M. Duhamel, Y. Beesetty, J. A. Mensah, O. Franken, E. Verbuggen, C. R. Fellbaum, G. A. Kowalchuk, M. M. Hart, A. Bago, T. M. Palmer, S. A. West, P. Vandenkoornhuyse, J. Jansa, and H. Bücking. 2011. "Reciprocal Rewards Stabilize Cooperation in the Mycorrhizal Symbiosis.” Science 333: 880-882.

Lederberg, J., and A. T. McCray. 2001. "'Ome Sweet 'Omics-A Genealogical Treasury of Words.” The Scientist 15: 8.

Lipsitch, M., S. Siller, and M. Nowak. 1996. "The Evolution of Virulence in Pathogens with Vertical and Horizontal Transmission." Evolution 50: 1729-1741.

Lloyd, E. 2017. "Holobionts as Units of Selection: Holobionts as Interactors, Reproducers, and Manifestors of adaptation." In Landscapes of Collectivity in the Life Sciences, edited by S. B. Gissis, E. Lamm, and A. Shavit, 351-367. London: The MIT Press.

Losos, J. 2011. Lizards in an Evolutionary Tree: Ecology and Adaptive Radiation of Anoles. Berkeley CA: University of California Press.

Mayo, D., and N. Gilinsky. 1987. “Models of Group Selection.” Philosophy of Science 54: 515-538. 
Mao, J., and T. Lu. 2016. "Population-Dynamic Modeling of Bacterial Horizontal Gene Transfer by Natural Transformation.” Biophysical Journal 110 (1): 258-68.

Margulis, L. 1991. "Symbiosis as a Source of Evolutionary Innovation: Speciation and Morphogenesis." In Symbiogenesis and Symbionticism, edited by L. Margulis and R. Fester, 1-14, Cambridge: MIT Press.

Maynard Smith, J. 1964. “Group Selection and Kin Selection.” Nature 201: 1145-1147.

McFall-Ngai, M., M. G. Hadfield, T. C. Bosch, H. V. Carey, T. Domazet-Loo, A. E. Douglas, N. Dubilier, et al. 2013. "Animals in a Bacterial World, A New Imperative for the Life Sciences." Proc. Natl. Acad. Sci. (USA) 110: 3229-3236.

Moran, N. A., and D. B. Sloan. 2015. “The Hologenome Concept: Helpful or Hollow?” PLoS Biol. 13 (12): e1002311.

Nash, D., T. Als, R. Maile, G. R. Jones, and J. J. Boomsma. 2008. "A Mosaic of Chemical Coevolution in a Large Blue Butterfly." Science 319: 88-90.

Okasha, S. 2005. "Multilevel Selection and the Major Transitions in Evolution." Philosophy of Science 72: 1013-1025.

Okasha, S. 2006. Evolution and the Levels of Selection. Oxford UK: Oxford University Press.

Osmanovic, D., D. A. Kessler, Y. Rabin, and Y. Soen. 2018. "Darwinian Selection of Host and Bacteria Supports Emergence of Lamarckian-like Adaptation of the System as a Whole." Biology Direct. doi:10.1186/s13062-018-0224-7.

OSTP (The White House Office of Science and Technology Policy). 2016. "Fact Sheet: Announcing the National Microbiome Initiative." United States Government. https://obamawhitehouse. archives.gov/the-press-office/2016/05/12/fact-sheet-announcing-national-microbiome-initiative. (Accessed December 10, 2018).

Queller, D. C., and J. E. Strassmann. 2016. "Problems of Multispecies Organisms: Endosymbionts to Holobionts.” Biol. Philos. 31: 855-873.

Rand, D. M., R. A. Hane, and A. J. Fry. 2004. "Cytonuclear Coevolution: the Genomics of Cooperation." Trends Ecol. Evol. 19: 645-653.

Rosenberg, E., O. Koren, L. Reshef, R. Efrony, and I. Zilber-Rosenberg. 2007. “The Role of Microorganisms in Coral Health, Disease and Evolution.” Nature Reviews Microbiology. 5(5): 355-62.

Rosenberg, E., and I. Zilber-Rosenberg. 2014. The Hologenome Concept: Human, Animal and Plant Microbiota, Springer International Publishing.

Rosenberg, E., and I. Zilber-Rosenberg. 2018. "The Hologenome Concept of Evolution After 10 Years." Microbiome 6: 78. doi:10.1186/s40168-018-0457-9.

Rosenberg, E., G. Sharon, and I. Zilber-Rosenberg. 2009. "The Hologenome Theory of Evolution Contains Lamarckian Aspects Within a Darwinian framework.” Environmental Microbiology 11: 2959-2962. doi:10.1111/j.1462-2920.2009.01995.x.

Roughgarden, J. 1983. "The Theory of Coevolution.” In Coevolution, edited by D. J. Futuyma and M. Slatkin, 33-64, Sunderland MA: Sinauer.

Roughgarden, J. 1995. Anolis Lizards of the Caribbean: Ecology, Evolution, and Plate Tectonics. Oxford UK: Oxford University Press.

Roughgarden, J. 2017. "Model of Holobiont Population Dynamics and Evolution: a Preliminary Sketch." In Landscapes of Collectivity in the Life Sciences, edited by S. B. Gissis, E. Lamm, and A. Shavit, 325-350. London: The MIT Press. 
Roughgarden, J. 2019. "Holobiont Evolution: Mathematical Model with Vertical vs. Horizontal Microbiome Transmission.” Preprint. bioR Riv. doi:10.1101/465310.

Roughgarden, J., S. F. Gilbert, E. Rosenberg, I. Zilber-Rosenberg, and E. Lloyd. 2018. "Holobionts as Units of Selection and a Model of Their Population Dynamics and Evolution." Biological Theory 13: 44-65. doi:10.1007/s13752-017-0287-1.

Schluter, J., and K. R. Foster. 2012. "The Evolution of Mutualism in Gut Microbiota via Host Epithelial Selection.” PLoS Biol 10(11):e1001424. doi:10.1371/journal.pbio.1001424.

Simon, B., J. Fletcher, and M. Doebeli. 2012. “Towards a General Theory of Group Selection.” Evolution 67(6): 1561-1572.

Skillings, D. 2016. "Holobionts and the Ecology of Organisms: Multi-species Communities or Integrated Individuals?" Biol. Philos. 31: 875-892.

Smith, J. 2007. “A Gene's-eye View of Symbiont Transmission.” Amer. Natur. 170: 542-550.

Trench, R. K. 1993. “Microalgal-Invertebrate Symbioses: A Review.” Endocytobiosis Cell Res. 9: 135175.

Turnbaugh, P. J., R. E. Ley, M. Hamady, C. M. Fraser-Liggett, R. Knight and J. I. Gordon. 2007. “The Human Microbiome Project." Nature 449: 804-810.

van Vilet, S., and M. Doebeli. 2019. "The Role of Multilevel Selection in Microbiome Evolution." Proc. Nat. Acad. Sci. (USA). doi:10.1073/pnas.1909790116.

West, S. A., E. T. Kiers, E. L. Simms, and R. F. Denison. 2002. "Sanctions and Mutualism Stability: Why do Rhizobia Fix Nitrogen?" Proceedings of the Royal Society of London B: Biological Sciences 269: 685-694.

Woese, C. R. 2004. “A New Biology for a New Century." Microbiology and Molecular Biology Reviews. 68(2): 173-86. PMID: 15187180.

Wright, S. 1931. "Evolution in Mendelian populations." Genetics 16: 97-159.

Yamamura, N. 1993. "Vertical Transmission and Evolution of Mutualism and Parasitism." Theor. Pop. Biol. 44: 95-109.

Zeng, Q. S. Wu, J. Sukumaran, and A. Rodrigo. 2017. "Models of Microbiome Evolution Incorporating Host and Microbial selection.” Microbiome 5(1): 127. doi:10.1186/s40168-017-0343-x.

Zilber-Rosenberg, I., and E. Rosenberg. 2008. "Role of Microorganisms in the Evolution of Animals and Plants: the Hologenome Theory of Evolution." FEMS Microbiology Revierws 32: 723-735. doi:10.1111/j.1574-6976.2008.00123.x.

(C) 2020 Author(s)

This is an open-access article distributed under the terms of the Creative Commons Attribution 4.0 International license, which permits anyone to download, copy, distribute, display, or adapt the text without asking for permission, provided that the creator(s) are given full credit.

ISSN 2475-3025 